\title{
ANÁLISIS DE ERRORES Y RETROALIMENTACIONES CORRECTIVAS EN INTERACCIONES ESPAÑOL- PORTUGUÉs MEDIANTE EL CONTEXTO
} TELETÁNDEM

\author{
Error Analysis and Corrective Feedback in Spanish-Portuguese-Language \\ INTERACTIONS IN Teletandem CONTEXTS
}

UNE ANALYSE DES ERREURS ET FEED-BACK DANS DES INTERACTIONS DANS LE PAIR ESPAGNOL-PORTUGAIS AU TÉLÉTANDEM

\author{
Consuelo Aponte Montejo \\ Especialización en Traducción, \\ Universidad del Valle. Profesora \\ auxiliar, Departamento de Lenguas \\ y Culturas Extranjeras. Grupo de \\ investigación en Lingüística Aplicada \\ (EILA), Universidad del Valle, Cali. \\ Escuela Ciencias del Lenguaje, \\ edificio E17, Ciudad universitaria, \\ Meléndez, Universidad del Valle, \\ Cali, Colombia. \\ consuelo.aponte@correounivalle.edu.co \\ https://orcid. \\ org/0000-0003-1498-6096
}

\begin{abstract}
Resumen
En lingüística aplicada, la enseñanza/aprendizaje de lenguas próximas se ha beneficiado del modelo de análisis de errores $(\mathrm{AE})$, sustentado en los modelos de análisis contrastivo (AC) y de interlengua. El objetivo del presente estudio de caso es analizarlos tipos deerrorescometidos por un estudianteuniversitariocolombianoen su aprendizaje de portugués como lengua extranjera (PLE), las retroalimentaciones correctivas (RC) más frecuentes durante sus interacciones con una estudiante brasileña en el contexto teletándem institucional integrado (TTDii, por su sigla en portugués) y observar las implicaciones de estos errores y retroalimentaciones en el proceso de aprendizaje. Es un estudio exploratorio de naturaleza etnográfica que incluye el modelo AE y su clasificación. Los resultados muestran una mejora de la competencia lingüística del estudiante probablemente como producto de su reflexión sobre el proceso de aprendizaje, en particular sobre los errores, que van disminuyendo en la medida que avanzan las interacciones.
\end{abstract}

Palabras claves: análisis de errores; portugués como lengua extranjera; enseñanza/aprendizaje de lenguas próximas; teletándem institucional integrado; TTDii, interlengua.

\section{Abstract}

In applied linguistics, teaching/learning of closely-related languages has benefited from the error-analysis model (EA), based on contrastive analysis (CA) and interlanguage approaches. This case study aims at analyzing the types of errors made by a Colombian university student in learning Portuguese as a foreign language $(\mathrm{PFL})$, as well as the most common corrective feedback ( $\mathrm{CF}$ ) he received when interacting with a Brazilian student in an institutional-integrated-teletandemcontext (TTDii, by its acronym in Portuguese), and at looking at the implications of this method in his learning process. This is an ethnographic exploratory study including the AE approach and its classification. Findings show improved linguis-

Received: 2017-07-26 / Accepted: 2018-08-27 / Published: 2019-09-15

doi: 10.17533/udea.ikala.v24n03a07 
tic competence by the student, probably as a result of reflection upon his learning process, particularly his errors, which were gradually decreasing as interactions moved forward.

Keywords: error analysis; learning Portuguese as foreign language; teaching and learning closely-related languages; institutional integrated teletandem context.

\section{Résumé}

En linguistique appliquée, l'enseignement/apprentissage des langues voisines a bénéficié du modèle de l'analyse d'erreur (AE) basé, à son tour, dans les modèles d'analyse contrastive (AC) et l'interlangue. Le but de cet article est d'analyser les types d'erreurs commises par un étudiant colombien dans son apprentissage de portugais comme langue étrangère (PLE), la rétroaction que a reçu au cours de ses interactions avec une étudiante brésilienne dans le contexte teletandem institutionnel intégré (TTDii, par son acronyme portugais) et observer ses implications dans le processus d'apprentissage. C'est étude exploratoire de caractère ethnographique qui comprend le modèle AE et sa classification. Les résultats montrent une amélioration de la compétence linguistique de l'étudiant sans doute comme un produit de sa réflexion sur le processus d'apprentissage, en particulier sur les erreurs, diminuant en avançant des interactions.

Mots-clés : analyse des erreurs; apprendre le portugais comme langue étrangère; enseignement et apprentissage des langues voisines; contexte teletandem institutionnel intégré. 


\section{Introducción}

En la enseñanza/aprendizaje de lenguas próximas y en particular de español y portugués, si bien la proximidad de las lenguas representa una ventaja en la fase inicial de comprensión, se convierte en una desventaja en la fase de producción al generar una falsa percepción de progreso en los estudiantes para quienes, como lo señala Almeida (1995) "las semejanzas pueden generar la sensación de que se facilitará en gran parte la tarea de aprendizaje" (p. 16), ${ }^{1}$ y por lo tanto, se empeñan poco en el reconocimiento de las diferencias entre las dos lenguas o exhiben la "tendencia a desconocer las diferencias entre elementos lingüísticos de la lengua de partida y la lengua objeto"2 (Almeida, 1995, p. 18). Esa percepción de facilidad implica, por un lado, más confianza y capacidad de riesgo en la comunicación, pero, por otro, "menor alerta ante las diferencias entre las lenguas, un menor grado de empeño en la tarea de aprendizaje y un exceso de autoconfianza que en última instancia suponen un freno al avance de la interlengua" (Alonso, 2005, p. 16).

Por consiguiente, es necesario buscar cómo estimular la reflexión en los estudiantes sobre los errores que cometen de manera sistemática, en su mayoría, como producto de la transferencia entre las lenguas próximas, los que, si bien no siempre interrumpen la comunicación, sí afectan una producción adecuada en la lengua que se aprende ya que, como afirma Camarena:

El papel que juega la lengua materna en el aprendizaje de una lengua extranjera próxima genera una interlengua particular que prácticamente no interfiere en el flujo comunicativo, pero que representa un tipo de errores que con frecuencia se fosilizan rápidamente. (Camarena, 2011, p. 71)

1 Las traducciones son mías. En el original: "as semelhanças podem suscitar a sensação de que a tarefa de aprendizagem vai ser em parte facilitada”.

2 En el original: "tendência de apagamento das distinções entre elementos linguísticos da língua de partida e a língua-alvo".
Adicionalmente, surge curiosidad por observar y estudiar el tratamiento de esos errores en cursos o programas diseñados para el aprendizaje de lenguas en contextos virtuales (por ejemplo, Livemocha, Busuu, Duolingo), dado que algunos de los estudiantes usuarios de dichas redes presentan mejor desempeño en sus producciones oral y escrita, como resultado de haber asumido un aprendizaje más autónomo. El trabajo con interacciones en ambientes virtuales y en particular en teletándem, contexto en el que se enmarca este estudio, podría mostrar diferencias significativas frente al trabajo con situaciones simuladas en el aula convencional.

Esta investigación busca responder a las siguientes preguntas, en el contexto teletándem institucional integrado (TTDii, por su sigla en portugués) entre una pareja de estudiantes universitarios, un colombiano y una brasileña:

- ¿Cuáles fueron los errores más frecuentes cometidos por el estudiante colombiano?

- ¿Cuáles fueron las retroalimentaciones correctivas (RC) más frecuentes empleadas por la estudiante brasileña?

- ¿Contribuyó el contexto TTDii a que el participante colombiano fuera consciente de su proceso de aprendizaje, en particular, de la producción de errores durante su proceso de aprendizaje de PLE?

Para responder estas preguntas, el presente estudio se propuso analizar los tipos de errores cometidos por el estudiante universitario colombiano en su proceso de aprendizaje de PLE y las retroalimentaciones correctivas más frecuentes empleadas por la estudiante brasileña durante sus interacciones en el contexto teletándem institucional integrado. Se partió del supuesto de que dicho contexto puede llevar al estudiante a un mejor desarrollo de la competencia lingüística en su aprendizaje de PLE, al minimizar la producción sistemática de errores, probablemente como consecuencia de la cercanía de las lenguas en cuestión, y al mismo tiempo a que sea consciente de su proceso de aprendizaje, un aspecto poco visible en el aula convencional. 


\section{Marco teórico}

\section{El error en el aprendizaje/enseñanza de PLE para hispanohablantes}

En lingüística aplicada, la enseñanza, el aprendizaje y la adquisición de lenguas próximas se ha beneficiado desde hace muchas décadas del modelo de análisis de errores ( $\mathrm{AE}$ ), que a su vez se sustenta en los modelos de análisis contrastivo (AC) y de interlengua. El primero fue iniciado por Charles C. Fries (1945) y reformulado por Pit Corder (1967), quien planteó el estudio sistemático de la producción del estudiante de lengua extranjera a partir de la lista de errores frecuentes para valorar su relevancia e indicar dificultades de aprendizaje. El segundo, presentado por Larry Selinker $(1972,1992)$, define y estudia el sistema lingüístico empleado por el estudiante de una segunda lengua, quien desempeña el papel de intermediario entre su lengua nativa y la lengua en aprendizaje.

En su estudio acerca de la producción del estudiante de lengua extranjera por medio de un inventario de los errores más frecuentes, Corder (1967) plantea una diferencia entre el concepto de error y el de falta o equivocación. Según él, el error es una desviación que aparece en la producción verbal del aprendiente de una segunda lengua o lengua extranjera como consecuencia del desconocimiento de la regla correcta (error sistemático), y se refiere a los términos falta o equivocación, como errores ocasionales que el aprendiz comete por lapsus o descuidos. Asimismo, propone la clasificación de los errores en dos grandes categorías:

1. Errores de interferencia o interlingüísticos, instancia desviada de la norma con respecto a la lengua que se aprende y que tiene lugar en la actuación del individuo como resultado de su familiaridad con las dos lenguas.

2. Errores intralingüísticos, que se originan dentro de la estructura interna de la lengua que el alumno estudia.
Por su parte, en sus reflexiones acerca del análisis de errores, Vásquez (2009, p. 114) considera que:

- Las faltas y los errores hacen parte del proceso de aprendizaje.

- Desde el punto de vista etiológico responden a varias causas, no solo a la distracción, la falta de esfuerzo, etcétera, sino, sobre todo, a la interferencia de la lengua materna y de otras lenguas extranjeras, así como a estrategias de regularización, simplificación y generalización.

- Los errores no se evitan a través de ciertas progresiones gramaticales o de otro tipo, ni siquiera a través de la corrección continua, propia o de quien enseña.

- Que un número considerable de errores responden a la formación de hipótesis que realizan las personas que aprenden.

En relación con la $\mathrm{RC}$, se encuentran estudios representativos ya sea sobre su definición como "una respuesta correctiva a la producción del aprendiz que no coincide con la lengua meta" (en Li, 2010, citado en Asanin, 2016, p. 309) o como la "respuesta que el profesor u otro aprendiente proporciona como reacción a un enunciado de otro aprendiente que contiene un error" (Ellis, 2005, citado en Asanin, 2016).

Este estudio se apoya en la tipología de RC de Lyster y $\operatorname{Ranta}^{3}$ (1997, p.48):

1. Corrección explícita: se proporciona al aprendiz la forma correcta indicando que lo que ha dicho es incorrecto;

2. Reformulación: se repite la producción incorrecta del aprendiz, cambiando las partes necesarias para convertirlo en una frase $u$ oración correcta.

3. Solicitud de aclaración: solicitud de aclaración de la producción del estudiante mediante preguntas.

3 En inglés: explicit correction, recast, clarification request, metalinguistic feedback, elicitation, repetition. 
4. Retroalimentación lingüística: contiene comentarios, información gramatical o preguntas relacionadas con la producción del aprendiz sin ofrecerle la forma correcta de manera explícita.

5. Inducción: se refiere a tres técnicas diferentes que los profesores utilizan para obtener la forma correcta del alumno: Primero, el profesor anima al aprendiz a que termine su frase. Segundo, el profesor utiliza preguntas para obtener la forma correcta del alumno. Y, por último, se pide al aprendiz que reformule lo dicho.

6. Repetición: se utiliza para comprobar si el aprendiz ha entendido lo que ha dicho el profesor.

Los postulados teóricos de los modelos mencionados han referenciado los estudios más representativos en lenguas próximas que en el caso de español y portugués en la bibliografía consultada fueron realizados por investigadores de Brasil (Almeida, 1995; Grannier, 2000; Grannier y Carvalho, 2001; España (Alonso, 2013), y México (Camarena (2011).

Como propuesta metodológica específica, Almeida (1995) ya planteaba en el título de su trabajo: Uma metodologia especifica para o ensino de linguas próximas? Más adelante, Grannier (2000) apoyó su propuesta en la producción escrita cuando no en la oral, consolidada ya en el siglo XX en los desarrollos en lingüística aplicada para la enseñanza de lenguas extranjeras. La aplicabilidad de la propuesta heterodoxa de Grannier (2000) fue elaborado más adelante por Grannier y Carvalho (2001) a partir de un estudio preliminar de los errores cometidos por estudiantes de portugués en su proceso de adquisición de la lengua, ofreciendo ejemplos de un proyecto de material didáctico, cuya finalidad es estimular el reconocimiento no solo de las semejanzas entre el español y el portugués, sino también de las diferencias entre ambas lenguas: el énfasis de su propuesta.

Natel (2002) plantea varios cuestionamientos sobre la manifestación de la fosilización, que van desde la identificación de su naturaleza, su inicio, el tiempo que persiste, hasta los aspectos de la interlengua influyentes en la aparición de dicha fosilización y las condiciones de su ocurrencia. Alonso (2013) examina la transferencia lexical como mecanismo de la lengua materna diferente de la interferencia y presenta sus implicaciones didácticas en el campo de la enseñanza de portugués para hispanohablantes.

Posteriormente, Almeida (2004) aborda los esfuerzos más recientes para plantear teorías que puedan representar una gran diferencia en la manera tradicional de enseñar PLE en contextos marcados por la presencia del español.

En su revisión de las propuestas metodológicas que se han venido haciendo en torno a las ventajas en el uso de los modelos AC y AE, Alonso justifica la importancia de presentarlos y discutirlos con los estudiantes desde el inicio del curso, así:

[...] al compartir esta estrategia contrastiva con el alumno le damos una herramienta que le permite utilizar procedimientos como la autocrítica de la producción de forma más eficaz. Ser conscientes de los puntos diferenciales nos permite hacer una revisión de nuestra producción que nos conducirá a localizar posibles errores que somos incapaces de detectar durante el proceso de producción. (Alonso, 2005, p. 34)

Igualmente, de gran relevancia en contextos de no inmersión, el trabajo de Camarena (2011) presenta el resultado del análisis de errores y fenómenos de interlengua producidos por estudiantes de portugués lengua extranjera en la Universidad Nacional Autónoma de México como parte de su investigación doctoral sobre la eficiencia en la enseñanza del portugués.

Sobre la RC, autores como Alonso (2015) han realizado trabajos sobre el efecto de la corrección en la expresión escrita en estudiantes hispanohablantes aprendices de portugués, profundizando sobre aspectos específicos y contribuyendo con la investigación en esta área.

De manera general, los estudios reseñados proponen el diseño de actividades de modalidad escrita mayoritariamente, ya que dan a los estudiantes el 
tiempo para reflexionar sobre el error y, al hacerlo, adquirir mayor consciencia sobre las diferencias de las lenguas en cuestión. Sin embargo, aunque estas propuestas contribuyen a enfrentar dificultades específicas a partir de errores estudiados, no dan cuenta de las actitudes y el desempeño de los estudiantes en un posible seguimiento hacia la superación de sus errores ya que como señala Vásquez: "Es necesario contar con herramientas que permitan al estudiantado evaluar su propio aprendizaje. $\mathrm{Si}$ en las personas jóvenes y adultas no existe una actitud consciente en cuanto al aprendizaje se puede afirmar que éste no será eficaz o no todo lo eficaz que podría ser” (Vázquez, 2009, pp. 113-120).

\section{Tándem y teletándem: una evolución del método}

El término tándem hace referencia a la bicicleta de doble asiento que requiere que sus ocupantes se muevan de manera sincronizada para desplazarse. En el campo del aprendizaje de lenguas, el tándem data de los años sesenta en Alemania y ha tenido amplia expansión desde entonces en otros países europeos como una modalidad alternativa y complementaria al aprendizaje formal de lenguas extranjeras (Vassallo y Telles, 2006).

Vassallo y Telles (2009) describen ampliamente los inicios de la metodología del tándem, las formas como se ha practicado y se practica actualmente: independiente, integrada o complementaria a un curso de lenguas, con o sin sesiones de mediación por un periodo de tiempo largo o corto; por pares (solo dos estudiantes) o por grupos - cuando interactúan estudiantes de dos cursos de diferentes lenguas localizados en dos países diferentes-.

Según los mismos autores, los principios que lo rigen, trazados en los años noventa con la creación de la Red Internacional de Tándem ${ }^{4}$ son:

4 Citada como una agrupación fundada como RiBo en 1992, por Helmut Brammerts, Ruhr-Universität Bochum.
- La separación de las lenguas o principio de igualdad: supone que se dedica el mismo tiempo para la práctica de cada una de las lenguas involucradas en el proceso. Eso garantiza que haya exposición balanceada en las dos lenguas.

- Reciprocidad: este principio está relacionado con el intercambio de los roles. Brammerts afirma que "la interdependencia mutua entre los dos interactuantes demanda igual compromiso, de manera que ambos se beneficien tanto como sea posible del trabajo en conjunto" (2003, p. 29).

- Autonomía: este principio está estrechamente relacionado con los dos anteriores, ya que las decisiones acerca de las interacciones pueden ser tomadas por los interactuantes de manera conjunta. Ellos tienen libertad para determinar los temas, materiales, cómo se realizará la corrección de los errores y los medios para lograr las metas establecidas.

Con el tiempo, el tándem se ha configurado como un término que designa una modalidad de aprendizaje abierta (no sigue un programa curricular fijo), intercultural (envuelve diferentes lenguas y culturas), interpersonal (se practica a dúo) y socialmente simétrica (hay alternancia de roles) (Benedetti, 2010).

Teletándem, el modelo de tándem adaptado por el profesor e investigador brasileño João Antonio Telles, difiere de las modalidades tándem que lo antecedieron por los recursos tecnológicos que utiliza, ya que los aplicativos Windows Live Messenger o Skype permiten la comunicación oral y textual (lectura y escritura) y la visualización de imágenes por medio de cámara web, en tiempo real. Este modelo se creó con el objetivo básico de ofrecer el tándem a alumnos universitarios brasileños que deseen aprender un idioma extranjero con estudiantes de otros países que estén aprendiendo portugués. 


\section{El error y la retroalimentación correctiva en teletándem}

No hay gran variedad de estudios sobre el error y la retroalimentación correctiva en teletándem en la bibliografía consultada. Gianini (2008), en su reflexión sobre la ocurrencia del error lingüístico en este contexto, destaca la importancia de la retroalimentación correctiva en ambientes virtuales, pues la considera una forma de ofrecer un retorno a las acciones relacionadas con el aprendizaje. Al analizar lo que cada interactuante hace con la corrección recibida, en términos de la gestión de su propio aprendizaje, Gianini concluye que la superación de los errores y, por consiguiente, el aprendizaje, dependerá de la adecuada gestión tanto en el ofrecimiento como en la recepción de la retroalimentación correctiva entre los interactuantes y afirma que la autonomía por sí sola no será efectiva en la superación de los problemas de aprendizaje si no hay reciprocidad:

El papel del "otro", del par más competente en las interacciones de teletándem es imprescindible, ya que es él quien proporciona la retroalimentación necesaria durante la interacción. De esa forma, el aprendizaje se construye en conjunto. De este modo, si no hay retorno, una respuesta/motivación a las acciones/actividades/expectativas de aprendizaje de ambas partes en sus respectivos momentos - como aprendiz o como profesorno ocurre el desarrollo del aprendizaje. ${ }^{5}$

Por su parte, al estudiar la forma como los participantes brasileños ofrecen RC a sus pares estadounidenses, Aranha y Cavalari (2015) muestran la tendencia a la corrección de la producción escrita de sus pares

5 En el original: "O papel do "outro" mais competente nas interações de teletándem é imprescindível, pois é ele quem provê o feedback necessário durante a interação. Assim, a aprendizagem se constrói em parceria e, em não havendo retorno (uma resposta/motivação às ações/ atividades/expectativa dos de aprendizagem de ambas as partes em seus respectivos momentos - quando professor ou quando aluno), não ocorre o desenvolvimento da aprendizagem." de teletándem de forma directa con atención a la forma lingüística y de acuerdo con sus creencias y experiencias como estudiantes.

\section{Metodología}

Con base en Souza (2006), esta investigación presenta características etnográficas, ya que "privilegia las documentaciones de los eventos sociales en sus contextos (mediante las grabaciones en audio y video, registro y observación de los participantes, etc.), así como la elaboración de la interpretación de sus significados para quienes están involucrados".6

Como lo señala Burns (1999), la grabación en audio y video es muy útil en la medida en que documenta detalles que pueden pasar desapercibidos en el momento de la interacción y pueden estar disponibles para su revisión.

Este estudio se realizó en el marco de un plan de colaboración entre la Escuela Ciencias del Lenguaje de la Universidad del Valle (Cali, Colombia) y el proyecto Teletándem Brasil Lenguas Extranjeras para todos ${ }^{7}$ de la Universidad Estadual Júlio Mesquita Filho -UNESP- (São Paulo, Brasil), siguiendo las características de la modalidad teletándem institucional complementaria (Aranha y Cavalari, 2014). En esta modalidad, el contexto tándem está incluido como un contenido en el programa del curso y por lo tanto es reconocido por la institución, en este caso, por el Departamento de Lenguas y Culturas Extranjeras de la Escuela Ciencias del Lenguaje de la Universidad del Valle. Al plantear el ejercicio, se hizo una presentación general del sistema a los estudiantes en la que se les explicaron: a) sus beneficios, en particular, los derivados de los principios que lo posibilitan y

6 En el original: "privilegia tanto documentações de eventos sociais em seus contextos típicos (através de gravações em áudio e vídeo, exame de artefatos socioculturais, registro e observação da participante, etc.) quanto a elaboração de interpretaçôes dos significados destes eventos para os agentes neles envolvidos." (p. 163).

7 Para información más detallada, ver "Sugerencias en español para teletándem en Teletándem Brasil Lenguas Extranjeras para todos", en http://www.teletandembrasil.org 
sustentan; b) los procedimientos básicos de una sesión de teletándem, entre los que se incluyen cómo emplear cada hora destinada a la sesión, sugerencias sobre revisión, corrección y elaboración de actividades entre una y otra sesión que favorezcan el aprendizaje colaborativo; c) la orientación pedagógica que tendrán las interacciones y que las diferenciará de las realizadas en un simple intercambio con un hablante nativo o en las redes sociales diseñadas para aprender lenguas en ambientes virtuales; d) el calendario de actividades relacionadas con los temas del curso para realizar semanalmente; e) la duración de las interacciones: tres meses con una sesión de una hora por semana, para un total de doce sesiones.

En relación con las técnicas y el procedimiento de recolección de datos, en este artículo se presenta el análisis de:

- Las grabaciones de cinco interacciones - dos de estas fueron realizadas por iniciativa de la pareja observada después de que la universidad brasileña entrara en paro- con un promedio de 50 minutos de duración ininterrumpida cada una, realizadas en el primer semestre académico del año 2016; las grabaciones se transcribieron en su totalidad ; para efectos del presente estudio se analizaron solo las transcripciones de las conversaciones sostenidas en portugués, con un promedio de 25 minutos de duración cada una.

- Las respuestas de los cuestionarios (Wallace, 1998; Gillham, 2000) aplicados al inicio y al final de las interacciones con el fin de indagar las concepciones sobre el error y las expectativas en relación con el aprendizaje de lenguas extranjeras mediante el uso del sistema teletándem. Los cuestionarios se adaptaron del cuestionario inicial de Cavalari (2009) y del cuestionario final de Bonfim (2014) (ver apéndices A y B).

Las interacciones grabadas con el programa Apowersoft ${ }^{8}$ se llevaron a cabo por Skype en el hora-

8 Grabador de pantalla gratis, aplicación web para capturar la pantalla de su PC. Puede capturar el video de la pantalla y rio regular de las clases y se almacenaron en los computadores de la sala de cómputo de la Escuela de Ciencias del Lenguaje.

De las doce interacciones programadas inicialmente solo seis pudieron llevarse a cabo, debido a que la universidad brasileña UNESP entró en paro como protesta ante la situación política de la presidenta del Brasil en ese momento (2016). De esas seis interacciones, la primera no se pudo grabar por fallas en el programa Hangouts ${ }^{9}$ sugerido inicialmente. La interacción fue utilizada en primer lugar para poner en contacto a los dos estudiantes y a las dos profesoras de las universidades participantes, y en segundo lugar, para verificar el funcionamiento y manejo de los equipos utilizados: computadores, programa de grabación y cámara.

Aunque los participantes pueden negociar el desarrollo de la sesión, de manera general, una sesión de teletándem tiene tres partes básicas:

1. Conversación sobre uno o varios temas en la que ambos interactuantes conversan sobre un tema sugerido. Se solicita ser buen oyente para responder a lo que el/la compañero/a dice, manteniendo la conversación y tomando, al mismo tiempo, apuntes sobre vocabulario, errores gramaticales y problemas de pronunciación, y considerar solo aquellos que impiden la comprensión.

2. Retroalimentación. Con base en las anotaciones tomadas sobre las posibles dificultades del/a compañero/a, concentrándose en explicaciones gramaticales. En el caso de no saberlas, ambos podrán despejar sus dudas, ya sea por medio de la consulta en internet o con profesores.

grabar con sonido todas las acciones que se realicen. Ver en https://www.apowersoft.es/grabador-de-pantalla-gratis.

9 Hangouts es una aplicación multiplataforma de mensajería instantánea desarrollada por Google Inc. Se creó para sustituir los servicios Google Talk, Google+ Messenger y Google+ Hangouts, unificando todos estos servicios en una única aplicación. 
3. Evaluación de la sesión. Los interactuantes dialogarán sobre cómo se sienten hablando en otro idioma, destacando los aspectos positivos e intentando prestar atención a las observaciones de posibles dificultades y las actitudes que ellas susciten.

\section{Participantes}

- Interactuante colombiano: veinticuatro años de edad, con español como lengua materna, estudiante del programa de Licenciatura de Música; matriculado en el curso Portugués I, ofrecido por la Escuela Ciencias del Lenguaje a estudiantes de todos los programas de la Universidad del Valle, con una intensidad horaria de cinco horas por semana. El participante firmó el formulario de consentimiento.

- Interactuante brasileña: veinte años de edad, con portugués como lengua materna, estudiante de la Facultad de letras en lengua portuguesa y una lengua extranjera, en este caso español, de la Universidad Estadual unESP de Brasil.

- La investigadora, quien observó directamente lo ocurrido y analizó los textos de las diferentes fuentes. ${ }^{10}$

Con el fin de preservar la identidad de los participantes, a cada estudiante se le asignó una letra inicial que refleja su nacionalidad: C (EC), para colombiano у в (ЕB), para brasileña.

\section{Análisis de los datos}

Se realizó una primera clasificación de errores en los niveles fonético-fonológico, morfosintáctico y lexical, adaptada para este estudio, siguiendo los criterios de Vázquez y Fernández (citados en Penadés, 2003). Posteriormente, se realizó una categorización de tipo de error para cada nivel lingüístico con el fin observar cuál tipo de error

10 En unesp, la profesora Kelly Cristiane Henschel Pobbe de Carvalho organizó las primeras tres interacciones en Brasil. presentaba más recurrencia. Cabe anotar que por no ser de interés en este estudio dar cuenta de las causas del error se indicarán algunas sin profundizar en ellas.

En un segundo momento y con el fin de observar los errores que más corrección generaron de la participante brasileña, se registraron los tipos de retroalimentación propuestos por dicha estudiante y se adoptó la tipología de Lyster y Ranta (1997) por considerarse completa para el objetivo del presente estudio.

Para codificar los datos, se utilizó un software de código abierto. ${ }^{11}$ Una vez recogidos, se introdujeron en tablas Excel para facilitar la visualización e identificar sus características sobresalientes mediante tablas y figuras.

Por último, en los cuestionarios inicial y final, aplicados antes y después de las interacciones, se seleccionaron las respuestas del estudiante colombiano, relacionadas con las concepciones sobre el error, y sus apreciaciones sobre la experiencia en su proceso de aprendizaje de PLE mediante el sistema TTDii.

\section{Resultados y discusión}

\section{Errores más frecuentes}

El número total de errores cometidos por el estudiante colombiano durante las cinco interacciones observadas fue de 279 , con un mayor número de aparición primero, en el aspecto lexical: 117 (41,9\% de todos los errores); segundo, en el aspecto morfosintáctico: $109(39,1 \%)$, seguido por el fonético-fonológico: 53 (19\%). Es importante señalar que, al inicio de las interacciones, el estudiante colombiano había cursado solo dos meses de clases de nivel básico. Sin embargo, en la Tabla 1 se puede apreciar una disminución significativa en la producción de errores a medida que avanzaron las interacciones.

11 OpenCode 4.02 es una herramienta para codificar datos cualitativos generados a partir de entrevistas, observaciones o notas. http://www.phmed.umu.se/enheter/ epidemiologi/forskning/open-code/ 


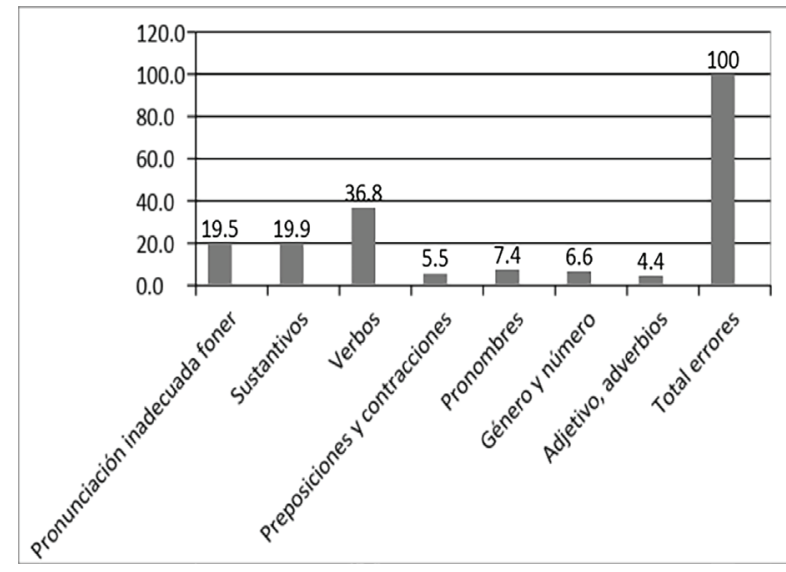

Figura 1 Categoría de errores

La Figura 1 muestra los tipos de errores clasificados en siete categorías con el número de ocurrencias. Sobresalen los errores en las categorías de verbos $(36,8 \%)$ en relación con el total de errores, seguidos de los errores en el uso de sustantivos $(19,9 \%)$ y de pronunciación inadecuada de fonemas (19,5\%). A continuación, se ofrecen algunos ejemplos, tal y como aparecen en las transcripciones de las grabaciones de las interacciones como ilustración de lo anterior:

Verbos: principalmente en el uso de tiempos pasados y flexiones:

(1) EC: Si, recentemente COMENCÉ. (La palabra correcta sería COMECEI).

EC: Por exemplo, em Panamá onde eu ESTUve. (La palabra correcta sería ESTIVE).
EC: Eu já FALE muito. (La palabra correcta sería FALEI).

Pronunciación inadecuada de fonemas:

(2) EC: Não é um mundo irreal?

(El estudiante pronuncia la consonante /1/ lateral alveolar propia de la fonética en español en lugar de pronunciarla vocalizada en / $\mathbf{u} /$ al final como suele hacerse en portugués brasileño).

EC: $\underline{\text { Vou a escrever. }}$

(El estudiante no pronuncia la consonante / $/$ / fricativa labio dental en portugués; pronuncia la $/ \mathbf{b} /$ oclusiva bilabial en español).

EC: Se refugiam na universidade.

(El estudiante pronuncia la consonante /r/ vibrante múltiple alveolar en español en lugar de pronunciarla fricativa velar o glotal sorda en portugués: / $\mathrm{b} /$ ).

Sustantivos: en su gran mayoría se presentan préstamos y lexemas con semas comunes pero no intercambiables en el contexto.

\section{Sustantivos:}

(3) EC: Em espanhol não estamos costumbrados com esses SONIDOS ...

(La palabra correcta sería sons).

EC: Você tem outra PREGUNTA?

(La palabra correcta sería PERGUNTA).

EC: Convido que você leia essa NOvela.

(La palabra correcta sería ROMANCE).

Tabla 1 Número de errores por interacción y por nivel lingüístico

\begin{tabular}{llllllll}
\hline Nivel lingüístico & $\begin{array}{c}\text { Interacción } \\
\mathbf{1}\end{array}$ & $\begin{array}{c}\text { Interacción } \\
\mathbf{2}\end{array}$ & $\begin{array}{c}\text { Interacción } \\
\mathbf{3}\end{array}$ & $\begin{array}{c}\text { Interacción } \\
\mathbf{4}\end{array}$ & $\begin{array}{c}\text { Interacción } \\
\mathbf{5}\end{array}$ & $\begin{array}{c}\text { Total errores y } \\
\text { \% por nivel }\end{array}$ \\
\hline Fonético-fonológico & 17 & 12 & 11 & 6 & 7 & $53(19,0 \%)$ \\
Morfosintáctico & 27 & 24 & 29 & 16 & 13 & $109(39,1 \%)$ \\
Léxico & & & & & & 117 \\
& 30 & 28 & 31 & 18 & 10 & $(41,9 \%)$ \\
Total errores & 74 & 64 & 71 & 40 & 30 & \\
& $(26,5 \%)$ & $(22,9 \%)$ & $(25,4 \%)$ & $(14,3 \%)$ & $(10,7 \%)$ & 279 \\
\hline
\end{tabular}




\section{Retroalimentaciones correctivas}

La Tabla 2 muestra los tipos de retroalimentación correctiva empleados por la participante brasileña clasificados según la tipología de (Lyster y Ranta, 1997), con un total de 122 correcciones, cifra que comparada con el número total de errores detectados (279) nos lleva a concluir que 157 errores no generaron ninguna corrección de parte de la participante brasileña.

De los seis tipos de retroalimentación correctiva sobresale la corrección explícita empleada 104 veces (85\%) y mayoritariamente en el nivel morfosintáctico 40 veces $(38,5 \%)$; seguida de la retroalimentación lingüística 15 veces (12,3\%) empleada mayoritariamente en el nivel fonético; de repetición 2 veces $(1,6 \%)$ y reformulación $1(0,8 \%)$. De lo anterior se deduce que, si bien hubo más correcciones explícitas en el nivel morfosintáctico, fue el nivel fonético el único que generó retroalimentación metalingüística. (Tabla 2).

A continuación, se presentan algunos ejemplos que ilustran las retroalimentaciones correctivas en los niveles fonético y morfosintáctico por ser los más corregidos por la participante brasileña:

Corrección explícita en el nivel morfosintáctico:

(1) EC: Foi um livro que chamou minha atenção porque eu HABÍA...
EB: eu TINHA. (La estudiante brasileña corrige).

(2) EC: Eu gostei muito do livro. Embora TARDEI muito em leerlo, não ler-lo.

EB: Embora DEMOREI muito. (La estudiante brasileña corrige).

(3) EC: Ele é uma pessoa muito famosa porque tem muito carisma, é muito aceitado pOR os JóvENES.

EB: PELOS JOVENS. (La estudiante brasileña corrige).

Retroalimentación lingüística en el nivel fonético:

(1) Interacción 2

EC: A ver, a ver, não sei...

EB: Vai ver o quê? (Ella corrige la pronunciación de la consonante /v/).

EC: É que nós escrevemos com "v" mas falamos /b/.

EB: Vocês não têm o som /v/? Mas você falou bem "escrevemos" agora, é esse "v" que você tem que falar. Esse som é muito importante para nós. Você faz assim - Ella muestra como debe colocar los dientes sobre los lábios para pronunciar de manera correcta el sonido.

EC: É que eu devo ser muito consciente dessa pronunciación para falar bem porque sino esqueço. Por exemplo, quando estou cantando sou mais consciente dessa pronunciacion. Por exemplo, se eu diz: vivir, voy, volver, esos casos sim lembro a pronunciação correta, mas normalmente esqueço a pronunciação. Normalmente a Cali, falamos assim, é problema da pronunciação da cidade. Acho que já havia falado isto com você, certo?

Tabla 2 Tipo de retroalimentación correctiva por tipo de error

\begin{tabular}{lllll}
\hline $\begin{array}{l}\text { Tipo de retroalimentación } \\
\text { correctiva }\end{array}$ & Fonético & Morfosintáctico & Lexical & $\begin{array}{l}\text { Total } \\
\text { retroalimentaciones } \\
\text { correctivas }\end{array}$ \\
\cline { 1 - 5 } & 28 & 40 & 36 & $104(85 \%)$ \\
\hline Corrección explícita & 0 & 1 & 0 & $1(0,8 \%)$ \\
\hline Reformulación & 0 & 0 & 0 & 0 \\
\hline Solicitud de aclaración & 12 & 2 & 1 & $15(12,3 \%)$ \\
\hline Retroalimentación metalingiústica & 0 & 0 & 0 & 0 \\
\hline Inducción & 0 & 0 & 2 & $2(1,6 \%)$ \\
\hline Repetición & 40 & 43 & 39 & 122 \\
\hline Total retroalimentaciones correctivas & 43 & & & \\
\hline
\end{tabular}


EB: Sim. Mas eu já escutei cantores que eles usavam um pouco o som [v] por que? Eu já escutei por exemplo, a Marcela Gandara, então ela faz esse som do [v] assim não muito forte mas...

EC: É porque eles, por exemplo, Marcela Gandara e outros cantantes que eu diz são mexicanos e não sei exatamente como é sua pronunciação justamente dessa letra mas acho que eles a pronunciam melhor que nós.

EB: Sim, mas, é que eu discordo do que você diz. É melhor você praticar, viu?

EC: Ok, tarefa. Vou melhorar — risas.

(2) Interacción 3

EC: A universidade Nacional com certeza ficaria em paro, em greve. Uma universidade muito importante a nível pedagógico. (El estudiante pronuncia la consonante / $\mathrm{x}$ / fricativa velar en español en lugar de /3/ fricativa alveolar en portugués).

EB: Claro, o nível pedagógico é muito importante, né? (Ella pronuncia correctamente los sonidos /v/ e /3). Lembra o link que te enviei sobre fonética? Esse aí é muito bom para estudar os sons do português que são diferentes.

EC: Tudo bem. Vou revisar.

(3) Interacción 4

EC: Isso. Se alguien me convida...

EB: alguém, como quem? (El estudiante colombiano pronuncia la letra $/ \mathrm{l} /$ en español y la estudiante brasileña la corrige como /w/).

EC: se alguém - él continúa pronunciando la palabra con el sonido de la /1/ como en español' /'alrjen/

EB: Alguém como eu? (Ella dice la frase pronunciando la palabra "alguien" correctamente en portugués -/ aw'ge $\mathrm{j} /$

EC: como diz?

EB: Alguém do Brasil? - /aw'ge $\mathrm{j} /$

EC: ah, Ok. /aw'ge $\sim$ j/ - finalmente él pronuncia la palabra correctamente en portugués.

EB: Treina bem esse /l/ como /w/. A pronúncia é muito importante. Você também deveria cantar palavras com /l/ e lembrar que o som é /w/. Por exemplo,
Brasil, real, etc. Vou te mandar um link ótimo sobre fonética e você me fala o que você achou disso, tá bom?

EC: Sim.

\section{Desarrollo de las interacciones}

Para responder a la tercera pregunta de este estudio, (¿Contribuyó el contexto TTDii a que el participante colombiano fuera consciente sobre su proceso de aprendizaje, en particular, sobre la producción de errores durante su proceso de aprendizaje de PLE?), primero, se observó de manera general el desarrollo de las interacciones y, segundo, se registraron las respuestas, de los cuestionarios inicial y final, del estudiante colombiano a preguntas relacionadas con su concepción del error y sobre las apreciaciones de su experiencia en el contexto TTDii.

En relación con el desarrollo de cada interacción los participantes observados se concentraron más en la primera parte de las tres sugeridas por el contexto TTDii (conversación, retroalimentación y evaluación), es decir en la conversación, alternando espontáneamente y no de manera programada las correcciones y las apreciaciones sobre el desempeño. Las interacciones se desarrollaron de manera cordial, respetando los turnos para hablary animándose mutuamente para mantener una comunicación continua; desde el inicio negociaron la forma de corrección de errores (inmediata) y los compromisos por fuera de cada interacción considerando sus intereses y necesidades; el par colombiano fue quien estuvo más atento al cambio de idioma y fue más propositivo en relación con la organización de las actividades; la estudiante brasileña por su parte, demostró más paciencia en sus comentarios en los momentos de corrección de errores y ofreció material complementario.

Lo anterior se ilustra con algunos ejemplos extraídos de las interacciones.

(1) EC: Y ya - risas- eso es lo que yo hago y ya tenemos que hablar en portugués... con paciencia (Interacción 1). 
Tabla 3 Desarrollo de las interacciones

\begin{tabular}{|c|c|c|c|c|c|}
\hline & Interacción 1 & Interacción 2 & Interacción 3 & Interacción 4 & Interacción 5 \\
\hline Fecha y duración & $\begin{array}{l}27 \text { de abril, } 2016 \\
47 \text { minutos }\end{array}$ & $\begin{array}{l}18 \text { de mayo, 2016; } \\
40 \text { minutos }\end{array}$ & $\begin{array}{l}15 \text { de junio, 2016; } 50 \\
\text { minutos }\end{array}$ & $\begin{array}{l}22 \text { de junio, 2016; } 54 \\
\text { minutos }\end{array}$ & $\begin{array}{l}29 \text { de junio, 2016; } 49 \\
\text { minutos }\end{array}$ \\
\hline Temas & $\begin{array}{l}\text { Presentación } \\
\text { personal: edad, } \\
\text { estudio, trabajo. }\end{array}$ & $\begin{array}{l}\text { Gustos personales: } \\
\text { libros y escritores. }\end{array}$ & $\begin{array}{l}\text { Situación de } \\
\text { universidades, } \\
\text { posibilidades de paro; } \\
\text { actividades de sus } \\
\text { cursos. }\end{array}$ & $\begin{array}{l}\text { Planes para } \\
\text { después de terminar } \\
\text { universidad; } \\
\text { estereotipos } \\
\text { culturales. }\end{array}$ & $\begin{array}{l}\text { Vacaciones, funerales, } \\
\text { gripa. }\end{array}$ \\
\hline Idiomas & $\begin{array}{l}\text { Inicia en español; } \\
\text { cambio a portugués } \\
\text { en el minuto } 20\end{array}$ & $\begin{array}{l}\text { Inicia en español; } \\
\text { cambio a } \\
\text { portugués en el } \\
\text { minuto } 12\end{array}$ & $\begin{array}{l}\text { Inicia en español; cambio } \\
\text { a portugués en el minuto } \\
20\end{array}$ & $\begin{array}{l}\text { Inicia en portugués; } \\
\text { cambio a español en } \\
\text { el minuto } 30\end{array}$ & $\begin{array}{l}\text { Inicia en español; cambio } \\
\text { a portugués en el minuto } \\
35\end{array}$ \\
\hline $\begin{array}{l}\text { Compromisos entre } \\
\text { cada interacción }\end{array}$ & $\begin{array}{l}\text { Envío de lista de } \\
\text { errores y propuesta } \\
\text { de tema para la } \\
\text { siguiente interacción. }\end{array}$ & $\begin{array}{l}\text { Envío de lista } \\
\text { de errores y } \\
\text { preguntas de } \\
\text { corrección. }\end{array}$ & $\begin{array}{l}\text { Envío del anteproyecto } \\
\text { de grado del integrante } \\
\text { colombiano para lectura } \\
\text { del par brasileño. } \\
\text { Propuesta de tema } \\
\text { siguiente interacción }\end{array}$ & $\begin{array}{l}\text { Envío de ideas de } \\
\text { integrante colombiano } \\
\text { para club en español. } \\
\text { Envío de enlace sobre } \\
\text { fonética de integrante } \\
\text { brasileña }\end{array}$ & $\begin{array}{l}\text { Última grabación } \\
\text { interrumpida por } \\
\text { problemas técnicos; sin } \\
\text { embargo, acordaron } \\
\text { seguir comunicándose } \\
\text { sin grabarse. }\end{array}$ \\
\hline
\end{tabular}

EC: Bueno entonces ahora yo te propongo que hablemos en portugués para que podamos hablar 25 minutos en portugués... (Interacción 2).

EC: Bueno entonces ahora vamos hablar en portugués. Comencé hablando español, ahora tú comienza en portugués, ¿vale? (Interacción 3).

(2) EC: Você pode escrever meus erros? Eu quero ter um registro das coisas a melhorar (Interacción 2).

EC: Tá bom... Eu vou para minha aula e eu espero que podamos continuar com nossas interações sem maior problema. A próxima interação você começa, lembra? (Interacción 3).

EC: Você tinha uma tarefa sobre o que vamos falar hoje (Interacción 4).

(3) EC: Calma, você está desesperado, calma. Eu também esqueço, vamos tentar de novo. Continua... (Interacción 1).

EC: Eu vou te enviar os nomes dos escritores para você pesquisar. (Interacción 2)

EC: Sim, por isso é que é difícil eu falar agora um livro pequeno. Mas esse da Lygia Bojunga que eu te falei e mandei por e-mail, ele é pequeno, bem pequeno. (Interacción 3).
La Tabla 3 presenta de manera resumida aspectos puntuales a lo largo del desarrollo de las interacciones, siendo muy importante el hecho de que estos fueran producto de las negociaciones de los dos participantes, con excepción de las dos primeras fechas para realizar las interacciones, que fueron programadas por las profesoras de las dos universidades participantes.

Respecto a la concepción del error y a las apreciaciones del estudiante colombiano sobre su experiencia en el contexto ITT, es relevante su definición del error antes y después de la experiencia, la declaración adicional sobre su deber como estudiante de autoevaluar su proceso de aprendizaje y la consciencia de la dificultad en su producción de la lengua extranjera frente a la facilidad de su comprensión. Asimismo, el reconocimiento de las contribuciones de su par brasileña en los diferentes aspectos del aprendizaje (error y correcciones; fortalecimiento de habilidades; actitudes y sentimientos; motivación y atención para estudiar de manera autónoma, etc.). (Ver Tabla 4. Se conservan los errores gramaticales cometidos por el estudiante en su redacción). 
Tabla 4 Respuestas cuestionarios inicial y final

\begin{tabular}{|c|c|}
\hline Cuestionario inicial & Cuestionario final \\
\hline $\begin{array}{l}\text { P6. Considerando la enseñanza/aprendizaje de una } \\
\text { lengua extranjera, ¿qué es el error para usted? }\end{array}$ & $\begin{array}{l}\text { Retome sus respuestas a las preguntas } 7 \text { y } 8 \text { del cuestionario inicial y } \\
\text { comente si sus concepciones sobre el error se confirmaron o se modificaron } \\
\text { después de su experiencia en teletándem. Cite ejemplos. }\end{array}$ \\
\hline $\begin{array}{l}\text { El error para mí es la oportunidad que tenemos de mejorar } \\
\text { al conocer nuestras debilidades. Creo que el temor a hablar } \\
\text { por equivocarse es un gran error y pensar que si se sabe la } \\
\text { gramática ya se puede hablar el idioma. Uno puede saber } \\
\text { la gramática y aun así equivocarse. }\end{array}$ & $\begin{array}{l}\text { No tengo muchos cambios en mi opinión acerca del error. Solo agregaría } \\
\text { que el estudiante debe ejercitar la habilidad de autoevaluar su proceso } \\
\text { de aprendizaje constantemente. Confieso que me di cuenta que con el } \\
\text { portugués, entender, entendía pero cuando tenía que hablar era muy difícil, } \\
\text { que tenía que estudiar. }\end{array}$ \\
\hline
\end{tabular}

\section{P7. ¿Se deben corregir los errores? ¿Cómo?}

Sí, porque al corregir el error aprendemos. Cada persona tiene su técnica. Equivocarnos es parte esencial de nuestras vidas, reconocer y aclarar los errores es clave en el proceso de aprendizaje, si soy recurrente en un error específico, esto me permite identificar el aspecto exacto en el que estoy fallando o que se me dificulta aprender. Cada cual tiene su técnica.

\section{P8. Si la respuesta a la pregunta anterior fue} afirmativa, ¿cuáles errores se deben corregir y cómo?
Continúo pensando que el error se debe corregir, pues cada que esto sucede, uno aprende. Por ejemplo, recuerdo que yo siempre decía: "Eso...". Gracias a que mi par me corrigió varias veces esta palabra, cada vez que yo quería decir "eso", venía a mi mente de inmediato la corrección que me hizo mi par: "isso". Así con muchas palabras. Chistoso: nos dimos cuenta [de] que un gesto es una buena forma de corrección.

Describa las formas en las que su par de teletándem contribuyó o podría haber contribuido más con su aprendizaje de portugués brasileño.

Mi par contribuyó de muchas maneras a que yo aprendiera mejor el portugués: Corrigiendo constantemente mi pronunciación. Fue muy paciente. Me brindó confianza para hablar sin temor de equivocarme, y me equivocaba mucho.

Me ayudó constantemente a mejorar mi ortografía.

A ver el portugués como una lengua que puedo usar a diario y no simplemente como algo que aprendo en la academia.

Hablar con mi par me hizo comprometerme a estudiar más y buscar más herramientas por mi cuenta para así poder tener una mejor conversación en cada interacción. Para mí fue importante practicar la pronunciación y estudiar los verbos y sobre todo buscar los verbos en pasado.

Después de la participación en el proyecto, ¿cómo define usted el sistema teletándem?

Después de las interacciones que hice puedo definirlo como una herramienta de comunicación que pone de acuerdo a dos personas ubicadas en países diferentes, para poder hablar y practicar la lengua extranjera que están aprendiendo. No puedo negar que llegué a pensar que con toda la tecnología que hoy día tenemos al alcance (celulares, computadores, tablets. etc.), teletándem no hace algo diferente a lo que podría hacer yo desde mi casa a través de Facebook, es decir, buscar a alguien de Brasil, solicitar amistad y comenzar a hablar y así practicar el idioma extranjero. Sin embargo, entendí que teletándem nos brinda la seguridad de que estamos hablando con personas reales y es totalmente transparente, por lo cual tenemos total confianza de abrir una amistad con el par internacional y al mismo tiempo aprender con más atención lo que hacemos. 
Tabla 5 Aumento de producción de oral en portugués y porcentaje de errores cometidos

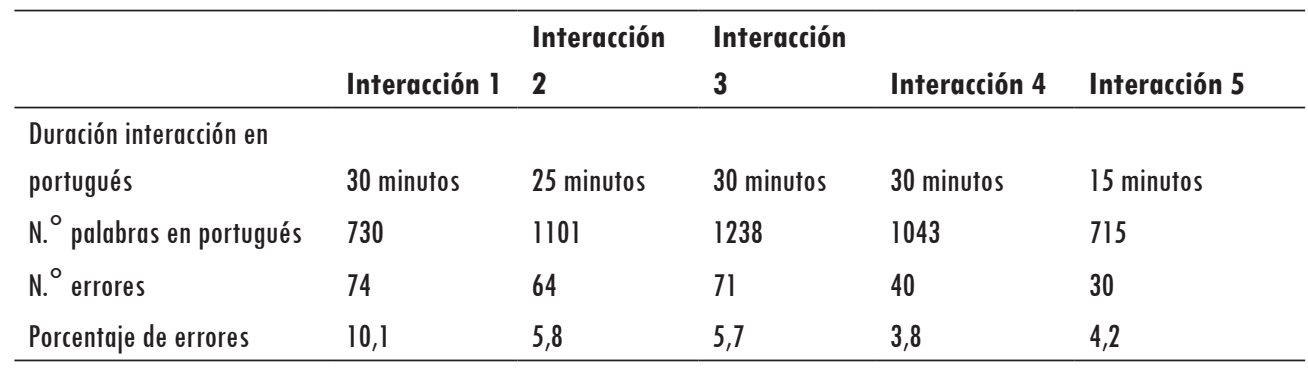

Finalmente, en la Tabla 5 se puede apreciar de una manera cuantitativa la contribución del contexto TTDii en la mejora del desarrollo de la competencia lingüística del estudiante colombiano al observarse mejor producción oral en portugués: los errores van disminuyendo a lo largo de las interacciones.

En las interacciones realizadas en el contexto teletándem institucional integrado (TTDii) entre una pareja de estudiantes universitarios, un colombiano y una brasileña Los resultados estudiados permitieron encontrar las siguientes respuestas a las tres preguntas formuladas.

\section{¿Cuáles fueron los errores más frecuentes cometidos por el estudiante colombiano?}

El mayor número de errores se cometieron en el nivel lexical 117 (41,9\% de todos los errores) en la categoría de los sustantivos con mayor ocurrencia de préstamos; seguido, del nivel morfosintáctico 109 (39,1\%), donde sobresalieron los errores en las flexiones y en el uso de tiempos verbales y, en tercer lugar, en el fonético-fonológico 53 (19\%) con pronunciación inadecuada de fonemas principalmente de las consonantes /v/, /l/, /g/ y /j/. En los tres niveles, como ya se ha mostrado en estudios anteriores en producción escrita, esos errores han sido clasificados como errores de transferencia debido a la cercanía de las lenguas (Grannier y Araújo, 2001; Camarena, 2011; Alonso Rey, 2014). Lo destacable aquí es que quien ha cometido esos errores ha sido un estudiante universitario colombiano, y por consiguiente, podrán orientar propuestas metodológicas y el diseño de recursos didácticos específicos para los cursos de la universidad.

\section{¿Cuáles fueron las retroalimentaciones correctivas (RC) más frecuentes empleadas por la estudiante brasileña?}

Sobresale la corrección explícita empleada 104 veces (85\%) y mayoritariamente en el nivel morfosintáctico 40 veces $(38,5 \%)$; seguida de la retroalimentación lingüística 15 veces (12,3\%) empleada esencialmente en el nivel fonético; de repetición 2 veces $(1,6 \%)$ y reformulación 1 (0,8\%). De lo anterior se puede deducir que si bien hubo más correcciones explícitas en el nivel morfosintáctico, fue el nivel fonético el que comprometió más la comunicación, pues generó retroalimentación metalingüística, es decir, información gramatical y comentarios sobre la producción del interactuante colombiano de parte de la participante brasileña. Dicha situación probablemente propició reflexiones y acciones en el estudiante colombiano que lo llevaron a tomar decisiones con implicaciones positivas en su proceso de aprendizaje, dada la rápida disminución de errores al final de las interacciones realizadas. Este dato es muy significativo para este estudio ya que la reflexión, en particular, sobre los errores fonéticos, es casi inexistente por parte de los aprendices en situaciones simuladas durante el aula convencional.

\section{¿Contribuyó el contexto TTDii a que el participante colombiano fuera consciente sobre su proceso de aprendizaje, en particular, sobre la producción de errores durante su proceso de aprendizaje de PLE?}

Por tratarse de un contexto que permite una interacción auténtica en la que se vive el uso real, simultáneo del lenguaje, los participantes en Teletándem si bien 
se sienten ansiosos o tímidos, también albergan muchas expectativas en relación con los dos roles que están dispuestos a desempeñar: el de aprendiz de una lengua extranjera y el de profesor o instructor de su lengua materna. Es en esa relación justamente de enseñanza/aprendizaje que los participantes deciden asumir que tiene lugar la reflexión sobre la importancia de los errores y su corrección; aspecto que en la clase convencional se expresa mayoritariamente de parte del profesor, una vez se inicia el ciclo de error y corrección durante la comunicación, pero no de parte del alumno y menos cuando se trata de lenguas próximas.

En ese sentido, los resultados de la pareja de participantes observada permite evidenciar una respuesta positiva a esta pregunta, considerando que el estudiante colombiano:

- Reflexionó sobre sus acciones ya que hubo cambios y mejorías en las sucesivas interacciones.

- Se mostró muy activo, pues se responsabilizó de su propio aprendizaje y el de su compañera: preparó las sesiones teniendo en cuenta los intereses y las necesidades de ambos. (Sugerencias de libros, vídeos, uso de Facebook', Whatsapp para envío de recursos y material).

- Respondió a las retroalimentaciones correctivas de su compañera durante el proceso interactivo, dando y esperando una respuesta de su par, respectivamente.

Lo anterior sustenta las ventajas de este contexto frente a otros sistemas de aprendizaje de lenguas virtuales y que pueden resumirse, para efectos de este estudio así: en este tipo de interacciones se aprende del compañero y con el compañero. Brammerts y Calvert, 2006 (citado en Asanin, 2016).

\section{Conclusiones}

En este trabajo se analizaron los errores cometidos por un estudiante universitario colombiano de portugués como lengua extranjera y las retroalimentaciones correctivas recibidas de parte de una estudiante brasileña durante sus interacciones en el contexto TT Dii. Los resultados revelan que dicho contexto es una herramienta complementaria efectiva para que sus participantes trabajen de forma consciente sobre los errores que cometen de manera sistemática, producto principalmente de la proximidad entre el español y el portugués.

De manera general, ese trabajo consciente no se visualiza en el aula convencional y por lo tanto, el aprendizaje de PLE se torna difícil de tratar. En estas interacciones los participantes pueden desarrollar la capacidad de reflexionar sobre su propio proceso de aprendizaje.

Desde el punto de vista investigativo el uso de este contexto también ofrece ventajas frente a otros contextos virtuales, puesto que con el consentimiento de los estudiantes se puede realizar investigación sobre aprendizaje de lenguas. No obstante, es imprescindible la organización entre las universidades participantes para llevar a buen término todo el proceso en lo que respecta a horarios, formación de pares y orientación general sobre el uso del sistema y en particular sugerencias sobre los temas de discusión antes de comenzar las interacciones. Igualmente, dado el tipo de estudio realizado, sería necesario ampliar la investigación con un mayor número de estudiantes para obtener resultados más representativos.

\section{Referencias}

Almeida Filho, J. C. P. (1995). Uma metodologia específca para o ensino de línguas próximas? En Português para estrangeiros. Interface com o espanhol (pp. 13-21). Campinas: Pontes.

Almeida Filho, J. C. P. (2004). Questões da interlíngua de aprendizes de português a partir ou com a interposição do espanhol (língua muito próxima). Português para falantes de espanhol/Portuguese for Spanish speakers (pp. 183-191). Campinas: Pontes.

Alonso Rey, R. (2005). El mito de la facilidad en el estudio del proceso de enseñanza/aprendizaje de PLE-HE. Estudios Portugueses, 4, 11-38.

Alonso Rey, R. (2013). La transferencia léxica en la comprensión auditiva en los aprendices de portugués hablantes 
de español. Recuperado de: http://www.ufvjm.edu. $\mathrm{br} / \mathrm{site} /$ revistamultidisciplinar/files/2011/09/ La-transferencia-léxica-en-la-comprensión-auditiva-Rocío-Rey-Espanha1.pdf

Alonso Rey, R. (2014). Dificultades en el aprendizaje de la morfologia verbal del português por hispanohablantes: el presente de indicativo. Linha D'Água, 27(1), 87-115. http://dx.doi.org/10.11606/issn.22364242.v27i1p87-115

Alonso Rey, R. (2015). La capacidad de corrección de errores y el feedback correctivo escrito: estudio de casos de aprendices de portugués hispanohablantes. TrabalhosemLinguistica Aplicada, 54(2),303-327.https:// dx.doi.org/10.1590/0103-18134608162681

Aranha, S. y Cavalari, S. M. S. (2014). A trajetória do projeto Teletándem Brasil: da modalidade institucional não-integrada à institucional integrada. The EsPecialist, 35(2), 70-88. Recuperado de: http://hdl.handle. net/11449/122298.

Aranha, S. y Cavalari, S. M. S. (2015). Institutional integrated teletándem: What have we been learning about writing and peer feedback? (part 3). DELTA: Documentação de Estudos em Linguistica Teórica e Aplicada, 31(3), 763-780. https://dx.doi. org/10.1590/0102-445039175922916369

Asanin, M. (2016). La retroalimentación correctiva entre pares en un intercambio etándem español-inglés a través de una wiki. Disertación doctoral, Universidad Autónoma de Madrid. Disponible en http://hdl.handle. net/10486/672487

Benedetti, A. M. (2010). Aplicaciones potenciales del contexto teletándem para el aprendizaje de lenguas extranjeras. Moderna språk, 104(1), 42-58.

Bonfim, M. V. (2014). A autonomia no contexto teletándem institucional integrado. Dissertação (mestrado) - Universidade Estadual Paulista Julio de Mesquita Filho, Instituto de Biociências, Letras e Ciências Exatas. Recuperado de http://hdl.handle.net/11449/122193.

Brammerts, H. (2003). Autonomous language learning in tandem: The development of a concept. En T. Lewis y L. Walker, Autonomous language learning in tandem (pp. 27-36). Londres: Academic Electronic Publications Ltd.

Burns, A. (1999). Collaborative Action Research for English language teachers. Cambridge: Cambridge University Press.

Camarena, Ortiz, E. D. (2011). Análisis de interlengua en un corpus de portugués escrito de estudiantes castellano hablantes universitarios. Revista de Lingüistica y Lenguas Aplicadas, 6, 71-82. http://doi. org/10.4995/rlyla.2011.894.

Corder, S. P. (1967). The significance of learner's errors. IRAL-International Review of Applied Linguistics in Language Teaching, 5(1-4), 161-170. https://doi. org/10.1515/iral.1967.5.1-4.161

Fries, C. (1945). Teaching and learning English as a foreign language. Ann Arbor: University of Michigan Press.

Gianini, F. A. (2008). Abordagem do erro no processo de ensino e aprendizagem de língua estrangeira em teletándem. Recuperado de: http://www.teletándembrasil.org/site/docs/GIANINI.pdf

Gillham, B. (2000). The research interview. Londres y Nueva York: Continuum.

Grannier, D. M. (2000). Uma proposta heterodoxa para o ensino de português a falantes de espanhol. En N. Júdice, Português para estrangeiros: perspectivas de quem ensina (pp. 1-12). Nitéroi: Intertexto.

Grannier, D. M., y Carvalho, E. A. (2001). Pontos críticos no ensino de português a falantes de espanhol: da observação do erro ao material didático. En Comunicación presentada en el IV Congresso da Sociedade Internacional de Português Língua Estrangeira (SIPLE). Universidade Federal de Rio de Janeiro, Rio de Janeiro, 10-11 de noviembre, 1998.

Lyster, R. y Ranta, L. (1997). Corrective feedback and learner uptake: Negotiation of form in communicative classrooms. Studies in Second Language Acquisition, 19, 37-66. https://doi.org/10.1017/S0272263197001034

Natel, T. B. T. (2002). La proximidad entre el portugués y el español, ¿facilita o dificulta el aprendizaje. Actas ASELE XIII, 825-832.

Penadés Martínez, I. (2003-2004). Las clasificaciones de errores lingüísticos en el marco del análisis de errores. Linred: Lingüistica en la Red, (1). Disponible en: http://hdl.handle.net/10017/24703

Selinker, L. (1972). Interlanguage. IRAL-International Review of Applied Linguistics in Language Teaching, 10(1-4), 209232. https://doi.org/10.1515/iral.1972.10.1-4.209

Selinker, L. (1992). Rediscovering interlanguage: Applied linguistics and language study. Serie Applied Linguistics and Language Study. Londres y Nueva York: Longman. https://doi.org/10.1515/iral.1972.10.1-4.209

Souza, R. A. (2006). Uma reflexão acerca da construção do conhecimento na investigação do ensino de línguas. Estudos Anglo-Americanos: ABRAPUI, (29-30), 163-184. 
Vassallo, M. L., y Telles, J. A. (2006). Foreign language learning in-tandem: Theoretical principles and research perspectives. The EsPecialist, 27(1), 83-118.

Vassallo, M. L. y Telles, J. A. (2009). "Ensino e aprendizagem de línguas em tandem: princípios teóricos e perspectivas de pesquisa”. En Telles, J. A. (org.), Teletanden: um contexto virtual, autônomo e colaborativo

\section{Apéndice A. Cuestionario inicial}

Estimado participante:

Esta encuesta hace parte de un proyecto de investigación institucional en lingüística aplicada. Las informaciones obtenidas por este medio serán analizadas sin mencionar los nombres de los participantes: la ética profesional se respetará y se mantendrá a lo largo del desarrollo de este trabajo.

Su contribución será indispensable para la realización de esta investigación. Gracias por su atención y disponibilidad.

Nombre:

Edad:

Para usted, ¿qué significa aprender una lengua extranjera?

Para usted, ¿cómo es un buen profesor de idiomas?

Para usted, ¿cómo es un buen alumno de idiomas?

¿Qué piensa del uso del computador en el proceso de enseñanza/aprendizaje de una lengua?

Marque los programas que utiliza con habilidad:

_ Microsoft Office Word

__ Microsoft Office Excel

_ Microsoft Office Power Point para aprendizagem de linguas estrangeiras no século XXI (pp. 21-42). Campinas: Pontes Editores.

Vázquez, G. (2009). Análisis de errores, el concepto de corrección y el desarrollo de la autonomía. Revista Nebrija de Lingüistica Aplicada, 5.

Wallace, M. (1998). Action research for language teachers. Cambridge: Cambridge University Press.

_ Navegadores en internet

_ Windows Media Player

_ Youtube

_ Windows Live Messenger

_ Skype

Otros programas que desee mencionar:

Considerando la enseñanza/aprendizaje de una lengua extranjera, ¿qué es el error para usted?

- ¿Se deben corregir los errores? ¿Por qué?

- Si la respuesta a la pregunta anterior fue afirmativa, ¿cuáles errores se deben corregir y cómo?

- ¿Para qué sirve la evaluación en el aprendizaje/ enseñanza de lenguas extranjeras?

- ¿Qué piensa de la interacción entre alumnos en una clase de lenguas extranjeras?

- ¿Cómo puede ayudar una interacción por Skype en la enseñanza/ aprendizaje de una lengua extranjera?

- ¿Qué entiende usted por autonomía en el proceso de aprendizaje de una lengua extranjera?

- Cite algunas actividades que considere autónomas para aprender una lengua extranjera en una interacción vía Skype? 
- ¿Qué espera de la experiencia de aprender y enseñar lenguas por medio del sistema teletándem a distancia?

- ¿Cree necesaria la ayuda de un profesor mediador para interactuar por Skype en el proceso de enseñanza/aprendizaje de una lengua extranjera? Justifique su respuesta.

\section{Apéndice B. Cuestionario final}

1. Después de la participación en el proyecto, ¿cómo define usted el sistema teletándem?

2. Según su experiencia, ¿cuáles serían las tres "reglas de oro" para una experiencia exitosa en teletándem?
3. Retome sus respuestas a las preguntas 2 y 3 del cuestionario inicial (CI) y comente los posibles cambios.

4. Describa las formas en las que su par de teletándem contribuyó o podría haber contribuido más con su aprendizaje de portugués brasileño.

5. Retome sus respuestas a las preguntas 7 y $8 \mathrm{del}$ cuestionario inicial (CI) y comente si sus concepciones sobre el error se confirmaron o se modificaron después de su experiencia en teletándem. Cite ejemplos.

6. Indique cuáles recursos utilizó en sus interacciones de teletándem y para qué los utilizó (use el cuadro siguiente).

\begin{tabular}{|c|c|c|c|c|}
\hline Recurso & $\begin{array}{l}\text { Utilizado con } \\
\text { frecuencia }\end{array}$ & $\begin{array}{l}\text { Utilizado } \\
\text { algunas veces }\end{array}$ & $\begin{array}{l}\text { Nunca } \\
\text { utilizado }\end{array}$ & $\begin{array}{l}\text { Utilizado } \\
\text { para qué }\end{array}$ \\
\hline \multicolumn{5}{|l|}{$\begin{array}{l}\text { Mecanismo de } \\
\text { traducción }\end{array}$} \\
\hline \multicolumn{5}{|l|}{ Libros de gramática } \\
\hline \multicolumn{5}{|l|}{ Redes sociales } \\
\hline \multicolumn{5}{|l|}{ Videos } \\
\hline \multicolumn{5}{|l|}{ Imágenes } \\
\hline \multicolumn{5}{|l|}{ Mapas } \\
\hline \multicolumn{5}{|l|}{ Gestos } \\
\hline \multicolumn{5}{|l|}{ libros de referencia } \\
\hline $\begin{array}{l}\text { Otro material (en } \\
\text { línea o en papel) }\end{array}$ & & & & \\
\hline
\end{tabular}

How to reference this article: Aponte-Montejo, Consuelo (2019). Análisis de errores y retroalimentaciones correctivas en interacciones español-portugués mediante el contexto teletándem. Íkala, Revista de Lenguaje y Cultura, 24(3), 555-573. DoI: 10.17533/udea.ikala.v24n03a07 